\title{
Metabolomics and Marker Based Accelerated Stability of Kanji and Ethanol Extract of its Main Ingredient, Daucus carota L. Roots
}

Latif $A^{*}$, Hussain K, Bukhari NI, Shafi $\mathrm{H}$ and Mazhar M

Pharmaceutical Chemistry, Allama lqbal Campus, University of the Punjab, Lahore, Punjab, Pakistan

\begin{abstract}
A self-fermented probiotic beverage Kanji-prepared from roots of Daucus carota L. subsp. sativus (Hoffm.) Arcang. var. vavilovii Mazk. (Apiaceae)-is widely consumed in many Asian countries due to a number of therapeutic claims. However, this beverage is only available for 2-3 months due to instability and availability of raw material. Therefore, to make this remedy available for longer period, the present study describes accelerated stability and different kinetic parameters of Kanji and ethanol extract of its major ingredient using metabolomics comparison and marker-based HPLC method. The samples were stored at three different conditions of temperature and relative humidity for a period of six months. UV-visible metabolomics fingerprints of both the samples taken at different time intervals compared to assess the stability under stress conditions. The same samples were then analyzed using HPLC method with florescent detection for the determination of ferulic acid contents which were used to determine kinetic parameters and predict shelf life at $25^{\circ} \mathrm{C}$. Metabolomics fingerprints comparison showed decrease in peak intensities and appearance of entirely different profiles in samples stored at high temperature and relative humidity. Based on ferulic acid contents, both the products followed zero order degradation. Ethanol extract was found to be having higher shelf life, activation energy and pre-exponential factor than that of the Kanji. The results of the present study indicate that Kanji manufacturers may use UV-visible metabolomics profiles for the assessment of stability and ethanol extract of roots of Daucus carota L. to make this beverage available throughout the year.
\end{abstract}

Keywords: Kanji; Self-fermented probiotic beverage; Stability; Metabolomics finger prints; Daucus carota L.

\section{Introduction}

Stability, the time wherein a drug retains its chemical integrity and labeled potency within the specified limits, is essential for patient's safety by assuring uniform dose of a product throughout the shelf life and avoiding economic loss due to selling an unstable product. Unlike modern medicine-well-defined chemically-herbal products stability testing needs a special protocol because such preparations are a mixture of many unknown chemical entities. These unknown chemical constituents may react with each other without any apparent change and may raise stability concerns [1-5].

Kanji is a self-fermented probiotic beverage-prepared from roots of Daucus carota L. (Black carrots) subsp. sativus (Hoffm.) Arcang. var. vavilovii Mazk. (Apiaceae)-is consumed widely to improve digestion and protect liver in early summer season in some Asian countries like Pakistan and India [5]. This beverage is prepared in homes as a household remedy and also sold by road-side vendors from February-May each year due to harvesting season of the plant. This beverage is prepared without any preservative and consumed within 7-8 days due to instability. Due to change of color and taste, this product is available throughout the year. However, the popularity of this product demands its availability for a longer period of time and for such purpose ethanol extract of roots of Daucus carota has been selected because of its certain propertiesyield, easy drying, organoleptic, physicochemical and pharmacological activities-which are comparable to that of Kanji [6]. The findings of the present study may be beneficial for Kanji manufacturers in producing stable product and fulfilling its registration requirements.

Most of the herbal products manufacturers particularly in under developed countries are not assigning shelf life to herbal preparations properly considering them stable until there is no physical change, which is not a scientific approach. The demand of stability data of herbal products is increasing day-by-day as sales of such products are being regulated like pharmaceuticals in many countries of the world.
Furthermore, stability data is being made mandatory for the registration of such preparations in many advance countries. It was reported in 2007 that by 2011 any herbal product possessing any medicinal claim required to be registered in European countries [2]. In Pakistan, recently health regulatory authority is also developing regulations for the registration of herbal and homeopathic products. This indicates the need of research in this area to provide easy approaches which can easily be adopted by less equipped manufacturers to produce stable herbal medicines and generate stability data for drugs regulatory authorities.

The stability testing of herbal products similar to that of modern medicines is a challenging task due to their consistently inconsistent and complex nature. The unavailability of analytical standards and methods of analyses are other difficulties in stability studies of herbal products. To achieve such goals, several approaches need to be undertaken. In the past decade, regarding herbal products a new concept emerged wherein such drugs are considered active substances in their entity, whether or not the therapeutic constituents are known. This concept has led to the development of an approach for stability testing of herbal products called metabolomics fingerprint profiling. Several spectroscopic and chromatographic techniques can be used to acquire fingerprints to assess stability of herbal products. But these fingerprints cannot be used to estimate kinetic parameters therefore; a marker-based analytical method is also needed. The markers have been

*Corresponding author: Abida Latif, Head of Department, Pharmaceutical Chemistry, Allama Iqbal Campus, University of the Punjab, Lahore, Punjab, Pakistan, Email: abidalatifanwar@gmail.com

Received July 01, 2018; Accepted July 13, 2018; Published July 20, 2018

Citation: Latif A, Hussain K, Bukhari NI, Shafi H, Mazhar M (2018) Metabolomics and Marker Based Accelerated Stability of Kanji and Ethanol Extract of its Main Ingredient, Daucus carota L. Roots. Pharm Anal Acta 9: 589. doi: 10.4172/2153 2435.1000589

Copyright: (C) 2018 Latif A, et al. This is an open-access article distributed unde the terms of the Creative Commons Attribution License, which permits unrestricted use, distribution, and reproduction in any medium, provided the original author and source are credited. 
classified into eight categories which can be used to maintain chemical constancy in products [3]. A marker(s) which is characteristic to plant and has pharmacological activity is better choice to determine kinetic parameters and predict shelf life like modern medicine.

The authors of this study isolated two components from black carrot root extract, ferulic acid and 6,4'-dihydroxy 3'-propen chalcone with \%age yield of $0.1 \%$ and $0.005 \%$ respectively. Therefore, ferulic acid was considered as major component for further study. Current study described two approaches-UV/Visible metabolomics fingerprint profiling and HPLC method-for the stability studies of Kanji and ethanol extract of roots of Daucus carota $\mathrm{L}$.

\section{Materials and Methods}

\section{Plant material}

The roots of the plant-Daucus carota L. subsp. sativus (Hoffm.) Arcang. var. vavilovii Mazk.-also called black carrots were purchased from local vegetable market in the month of March. The material was authenticated by Professor Dr. Zaheer ud Din Khan, Department of Botany, Government College University, Lahore, Pakistan; wherein a voucher specimen was deposited vide reference No. G. C. Bot. Herb. 958. The roots were rinsed in tap water to remove extraneous matter and the residual water was evaporated.

About $1 \mathrm{~kg}$ of dried roots was crushed and $500 \mathrm{~g}$ of this material was macerated in $1.0 \mathrm{~L}$ ethanol at room temperature for $8 \mathrm{~h}$ with occasional shaking. Then the extract was collected and the residue was again extracted with $1.0 \mathrm{~L}$ of ethanol. Both the extracts were pooled and dried in vacuum at $40^{\circ} \mathrm{C}$.

\section{Preparation of fermented beverage (Kanji)}

The Kanji was prepared using the most commonly used traditional recipe as $113 \mathrm{~g}$ vertically sliced thin-long pieces of roots and $5 \mathrm{~g}$ each of red chilies, mustard seeds and table salt (sodium chloride) were added in a glass jar containing $1.50 \mathrm{~L}$ water. The jar was covered and the contents were allowed to ferment spontaneously at room temperature for 4 days $[7,8]$. Afterwards, the Kanji was stored at $10^{\circ} \mathrm{C}-15^{\circ} \mathrm{C}$ until used. The red chillies, mustard seeds and salt are used to impart good taste and color to the Kanji.

Ultraviolet/visible spectroscopy: The samples were scanned in ultraviolet and visible region using UV/Visible spectrophotometer equipped with UV-Probe operating system (Shimadzu Scientific Instruments, USA).

High performance liquid chromatography (HPLC): Chromatographic analysis was performed using liquid chromatography system 1200 series (Agilent Technologies, Waldronn, Germany) equipped with isocratic pump (G1310 A), auto sampler (G1329 A), column oven (G1316 A), DAD (G 1315 B) and florescent detector (G1321 A). The data acquisition was carried out using ChemStation, version A. 08.03 .

\section{Stability study protocol}

The study was performed using protocol described by International Conference on Harmonization (ICH) as suggested by Working Party of Herbal Medicinal Products of the European Agency for the Evaluation of Medicinal Products [4,9]. Kanji and ethanol extract were kept in screw caped transparent glass bottles and exposed for a period of 6 months to three different conditions of temperatures and relative humidity (Table 1). The humidity was controlled by saturated salt

\begin{tabular}{|c|c|c|}
\hline Temperature ( $\left.{ }^{\circ} \mathbf{C}\right)$ & Relative humidity (RH \%) & Saturated salt solution \\
\hline $30 \pm 2$ & $65 \pm 5$ & $\mathrm{NaCl}$ \\
\hline $40 \pm 2$ & $75 \pm 5$ & $\mathrm{NaCl}$ \\
\hline $60 \pm 2$ & $85 \pm 5$ & $\mathrm{KCl}$ \\
\hline
\end{tabular}

Table 1: Storage conditions for stability studies of Kanji and ethanol extract of roots of Daucus carota.

solution [10-13]. The samples were withdrawn and analyzed at 0 month (before starting experiment), 1, 2, 4 and 6 months.

\section{Preparation of samples for metabolomics fingerprints}

Kanji having concentration $1.0 \mathrm{mg} / \mathrm{ml}$ of ferulic acid was taken as a stock solution. The working sample solution having concentration 0.1 $\mathrm{mg} / \mathrm{ml}$ of ferulic acid was prepared by diluting the stock solution in ethanol.

The stock solution of ethanol extract having concentration $1.0 \mathrm{mg} /$ $\mathrm{ml}$ of ferulic acid was prepared in ethanol. The working sample solution having concentration $0.1 \mathrm{mg} / \mathrm{ml}$ of ferulic acid was prepared by diluting the stock solution with ethanol.

\section{UV-Visible spectroscopy}

The working sample solutions of Kanji and ethanol extract were scanned in UV-visible range $(200-800 \mathrm{~nm})$ using ethanol as a blank. The UV-visible profiles of samples taken at different time intervals from different storage conditions were compared to observe metabolomics changes.

\section{Preparation of samples for HPLC}

Samples of Kanji taken from different storage conditions at different time intervals were freeze-dried in order to avoid deterioration. The dried Kanji was then dissolved in HPLC grade methanol to get sample solution having concentration $5 \mathrm{mg} / \mathrm{ml}$. Ethanol extract taken from various storage conditions was also dissolved in in HPLC grade methanol to get sample solution having concentration $5 \mathrm{mg} / \mathrm{ml}$.

\section{Preparation of standard solutions for HPLC}

A stock solution of ferulic acid having concentration $1.0 \mathrm{mg} / \mathrm{ml}$ was prepared in HPLC grade methanol. Then a range of working standard solutions $(10.0,20.0,40.0,60.0,80.0,100.0$ and $120.0 \mu \mathrm{g} / \mathrm{ml})$ was prepared by diluting the stock solution with methanol.

\section{Chromatographic conditions}

All the samples and standard solutions for were filtered using 0.45 $\mu \mathrm{m}$ PTFE syringe filters (Whatman, Maidstone, England). Each sample/ standard solution $(20 \mu \mathrm{L})$ was eluted through column-Eclipse X DB-C18 ( $5 \mu \mathrm{m}, 4.6 \mathrm{X} 1500 \mathrm{~mm})$-using isocratic mobile phase comprising water: methanol: phosphoric acid (300:200:2.5, v/v/v) at flow rate of $1.0 \mathrm{ml} /$ min. The column temperature was maintained at $25^{\circ} \mathrm{C}$ and fluorescent detection was performed at $250 \mathrm{~nm}$ excitation and $410 \mathrm{~nm}$ emission wavelengths. The peaks were identified by comparing retention time to that of the standard. Calibration curve was constructed between concentration and peak area for the quantification of the ferulic acid in samples.

\section{Determination of kinetic parameters}

The order of the reaction was determined using graphical method $[14,15]$. For each storage temperature, graphs were plotted between $\%$ concentration and time, $\operatorname{lnK}$ and time, and reciprocal of concentration and time for zero order, first order and second order, respectively. The 
correlation coefficient of each of the graphs was evaluated and the plot with the best linearity was taken as order of the chemical reaction.

The slope of the plot having the best linearity was taken a rate constant $(\mathrm{K})$ at each storage temperature. Then the rate constants corresponding to three storage temperatures were converted in natural logarithm and the storage temperature in Kelvin is converted into reciprocal $(1 / \mathrm{T})$. Then a plot was constructed between $\operatorname{lnK}$ and $1 / \mathrm{T}$ to get linear regression equation for the determination of $\mathrm{K}$ at $25^{\circ} \mathrm{C}$, activation energy (Ea) and frequency constant (A). The slope of this plot corresponds to Ea/R (universal gas constant, $8.314 \mathrm{~J}$. mole ${ }^{-1}$. $\mathrm{K}^{-1}$ ).

Shelf life $\left(t_{90}\right)$ depends on the order of the reaction and can be calculated using different equations. For compounds following zero order degradation, a factor 0.105 is divided by rate constant to calculate the shelf life.

\section{Data analysis}

The samples were analyzed in triplicate and the results were averaged and expressed as mean $\pm \mathrm{SD}$.

\section{Results and Discussion}

The accelerated stability of Kanji and ethanol extract of roots of its main ingredient, Daucus carota L. was determined using two methods such as metabolomics fingerprint profiling and active marker quantification. Both the samples were kept at different storage conditions such as $30^{\circ} \mathrm{C} / 65 \% \mathrm{RH}, 40^{\circ} \mathrm{C} / 75 \% \mathrm{RH}$ and $60^{\circ} \mathrm{C} / 85 \% \mathrm{RH}$ [4]. The samples drawn at different time intervals were analyzed to get metabolomics fingerprints using UV-visible spectroscopy and determination of contents of ferulic acid by HPLC.

\section{Stability using metabolomics fingerprints profiling}

The result of the effect of temperature and relative humidity $\left(30^{\circ} \mathrm{C} / 65 \% \mathrm{RH}\right)$ on Kanji with the passage of time intervals is given in Figure 1. The metabolomics profiles of Kanji obtained from UVvisible spectroscopy at different time intervals indicated the gradual change in chemical composition. The profile obtained at time 0 month (pre-storage) indicated two peaks in UV region having maximum absorbance at 287 and $325 \mathrm{~nm}$. On the other hand, profiles obtained after 1, 2, 4 and 6 months indicated decrease in peak height at both the wavelengths. Besides this decrease in peak height, the gradual shift of peaks was also observed; peak at $287 \mathrm{~nm}$ was shifted to $278 \mathrm{~nm}$ and peak at $325 \mathrm{~nm}$ was shifted to $315 \mathrm{~nm}$. The metabolomics profile of Kanji sample obtained after 6 months storage was interesting because the peak height at wavelength $325 \mathrm{~nm}$ was greatly reduced and there was appearance of an additional peak at $224 \mathrm{~nm}$. These results indicated that Kanji was not stable at room temperature.

The result of the effect of storage of Kanji at $40^{\circ} \mathrm{C} / 75 \% \mathrm{RH}$ for six months is given in Figure 2. The metabolomics profiles of Kanji obtained from UV-visible spectroscopy at different time intervals indicated the gradual change in chemical profile. After 1 month there was decrease in peak height as well as shift in wavelengths as observed in case of Kanji stored at $30^{\circ} \mathrm{C}$. At $40^{\circ} \mathrm{C}$, the third peak appeared at $224 \mathrm{~nm}$, which was noted after six months in case of storage at $30^{\circ} \mathrm{C}$. The profile obtained after 2 and 4 months indicated that the peak at $325 \mathrm{~nm}$ disappeared and there was increase in height of peaks appearing at 265 and $224 \mathrm{~nm}$. The profile obtained after six months showed the decrease in height of all the three peaks. These results indicated that rate of chemical changes increased at $40^{\circ} \mathrm{C}$.

The results of the effect of storage of Kanji at $60^{\circ} \mathrm{C} / 85 \% \mathrm{RH}$ for

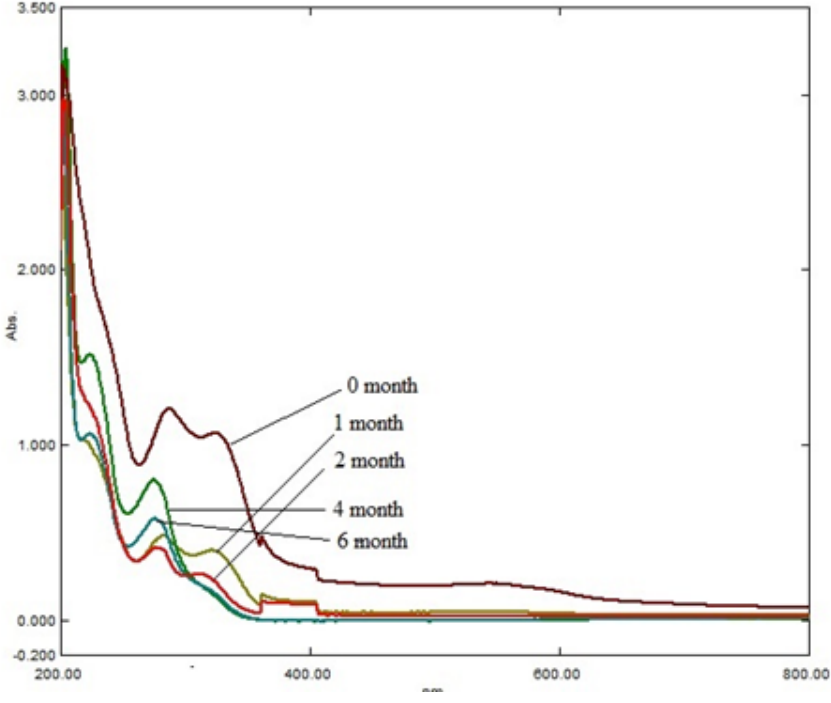

Figure 1: The overlay metabolomics profiles (UV-visible) of Kanji stored at $30^{\circ} \mathrm{C} / 65 \% \mathrm{RH}$ for six months, $\mathrm{RH}$ (relative humidity).

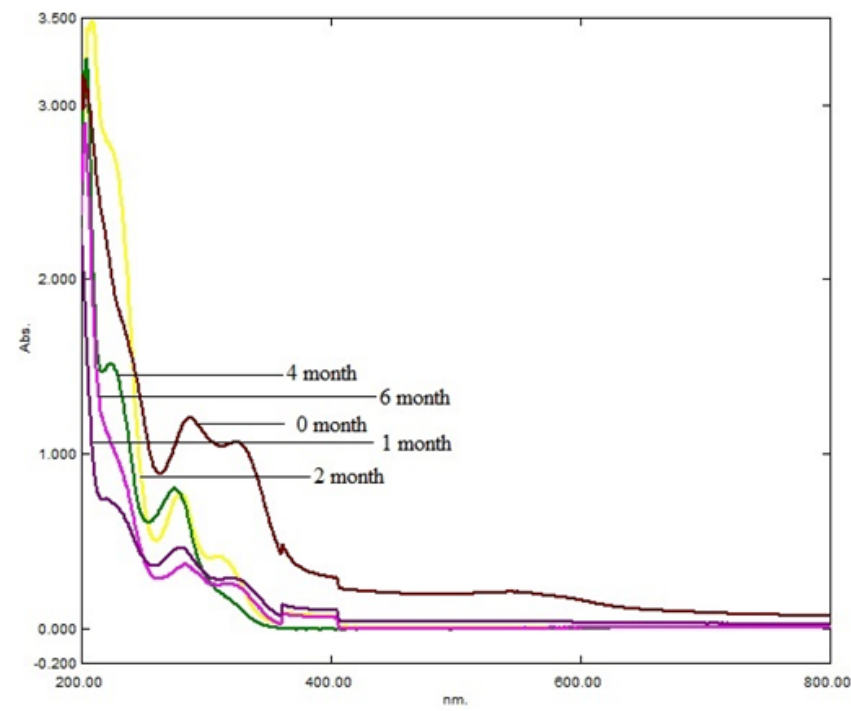

Figure 2: The overlay metabolomics profiles (UV-visible) of Kanji stored at $40^{\circ} \mathrm{C} / 75 \% \mathrm{RH}$ for six months, $\mathrm{RH}$ (relative humidity).

six months are given in Figure 3. The metabolomics profiles of Kanji obtained from UV-visible spectroscopy at different time intervals indicated the gradual change in chemical profile. After a month's storage, the peak appearing at $325 \mathrm{~nm}$ was shifted to lower wavelength but the height was slightly increased. On the other hand, the peak noticed at $287 \mathrm{~nm}$ was also shifted to lower wavelength but the height was decreased. On following months, 2 and 4, heights of both the peaks decreased. Again, the profile obtained after six months showed a very different look; two very big peaks appeared at 373 and $254 \mathrm{~nm}$. These results indicated further increase in chemical changes in Kanji.

The results of stability of ethanol extract stored at $30^{\circ} \mathrm{C} / 65 \% \mathrm{RH}$, $40^{\circ} \mathrm{C} / 75 \% \mathrm{RH}$ and $60^{\circ} \mathrm{C} / 85 \% \mathrm{RH}$ are given in Figures $4-6$, respectively. These results showed gradual decrease in peak heights with the passage of time at all the storage conditions. However, the profile of samples 


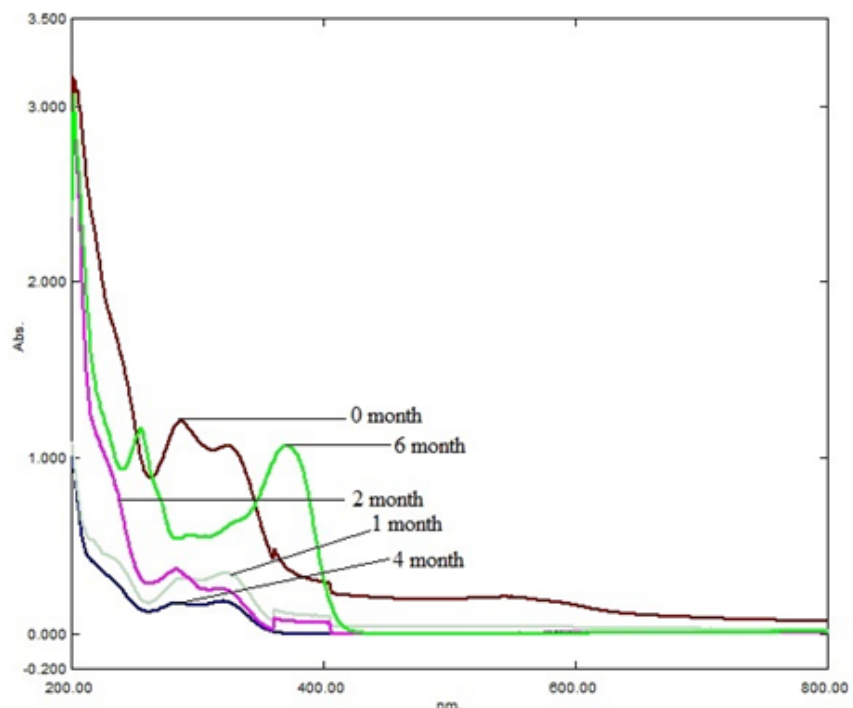

Figure 3: The overlay metabolomics profiles (UV-visible) of Kanji stored at $60^{\circ} \mathrm{C} / 85 \% \mathrm{RH}$ for six months, $\mathrm{RH}$ (relative humidity).

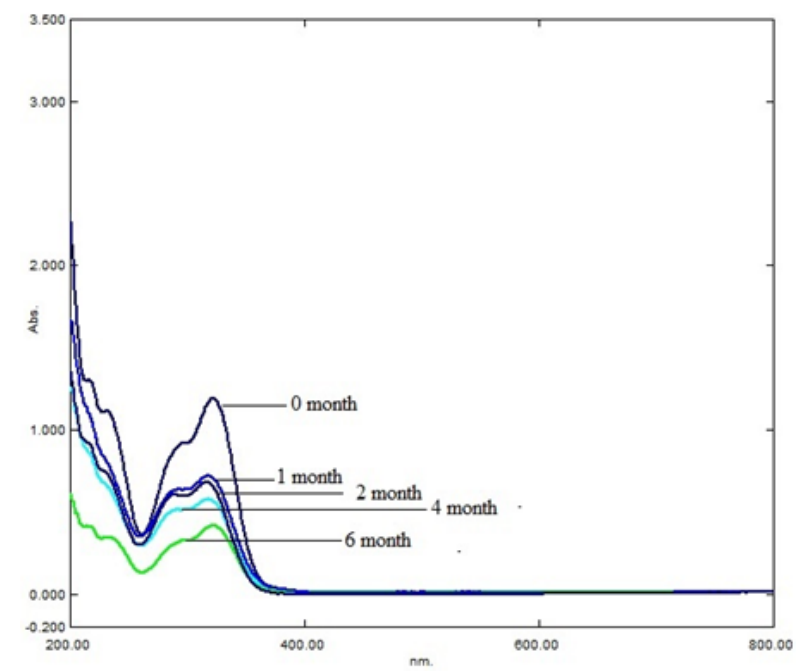

Figure 4: The overlay metabolomics profiles (UV-visible) of ethanol extract of roots of Daucus carota L. stored at $30^{\circ} \mathrm{C} / 65 \% \mathrm{RH}$ for six months, $\mathrm{RH}$ (relative humidity)

remained the same except the sample stored at $60^{\circ} \mathrm{C}$ and analyzed after 6 months. It indicated that at higher temperature, beside decrease in chemical there was development of metabolites that were having entirely different profile. The behavior of Kanji was also similar to that of the ethanol extract when stored at $60^{\circ} \mathrm{C}$ for six months. The change of Kanji profiles was faster than that of the ethanol extract. The possible reasons may be the water contents in Kanji as it was stored in the form of liquid and presence of additional ingredients.

The metabolomics fingerprint profile comparison of Kanji and ethanol extract samples stored at different temperatures and relative humidity indicated that the chemical constituents changed with the passage of time and the rate of chemical changes was found to be increasing at higher temperature. It was noted that after six months storage at $60^{\circ} \mathrm{C}$, the chemical nature of the samples was totally changed than that of the samples analyzed before storage.

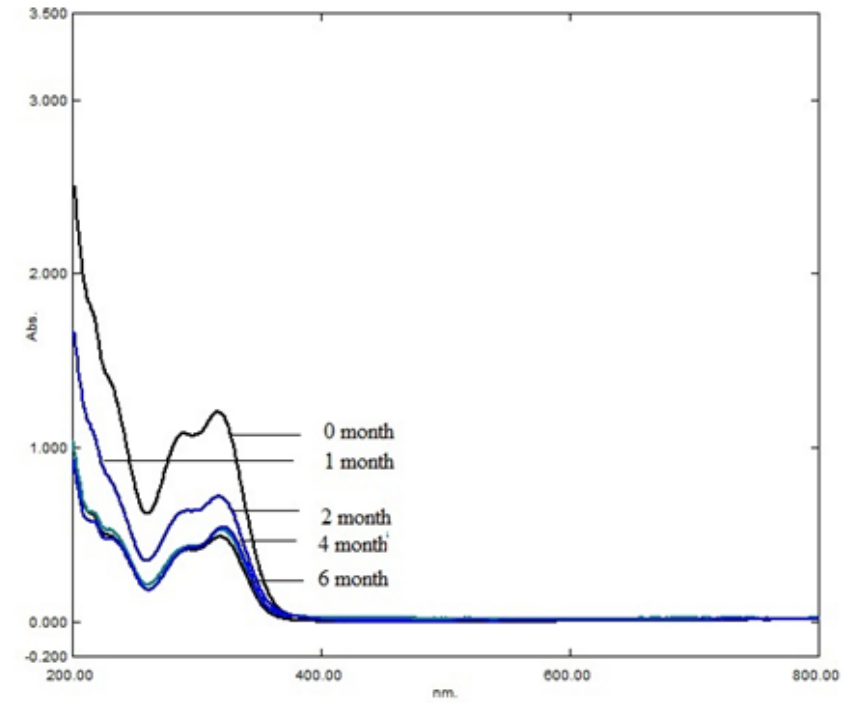

Figure 5: The overlay metabolomics profiles (UV-visible) of ethanol extract of roots of Daucus carota L. stored at $40^{\circ} \mathrm{C} / 75 \% \mathrm{RH}$ for six months, $\mathrm{RH}$ (relative humidity).

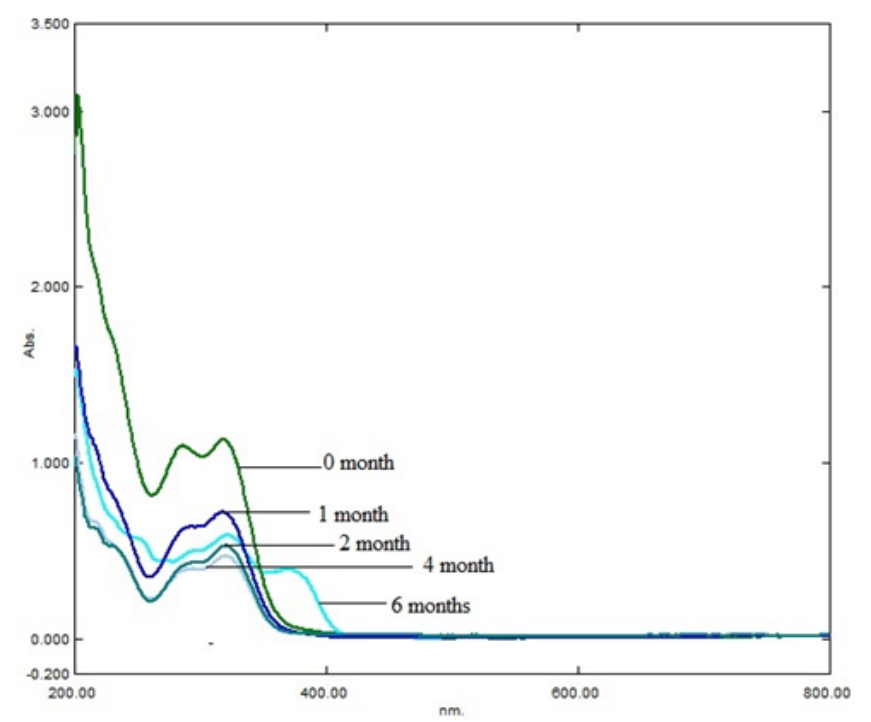

Figure 6: The overlay metabolomics profiles (UV-visible) of ethanol extract of roots of Daucus carota L. stored at $60^{\circ} \mathrm{C} / 85 \% \mathrm{RH}$ for six months, $\mathrm{RH}$ (relative humidity).

\section{Marker-based stability}

The samples taken at different time intervals from various storage conditions were analyzed by a validated HPLC method for the determination of ferulic acid. The calibration curves were constructed between concentration and peak area. The linear regression equation obtained from a calibration curve used for the determination of stability of Kanji with reference to ferulic acid was $\mathrm{Y}=0.4776 \mathrm{X}+1.9905$ with $\mathrm{R}^{2}=0.9957$, whereas the linear regression equation used for stability testing of ethanol extract was $\mathrm{Y}=0.5571+0.359$ with $\mathrm{R}^{2}=0.9957$. The chromatograms of standard and some of the samples are given in Figure 7.

\section{Percentage remaining of ferulic acid in samples}

The results of \% age remaining of ferulic acid in Kanji and ethanol 


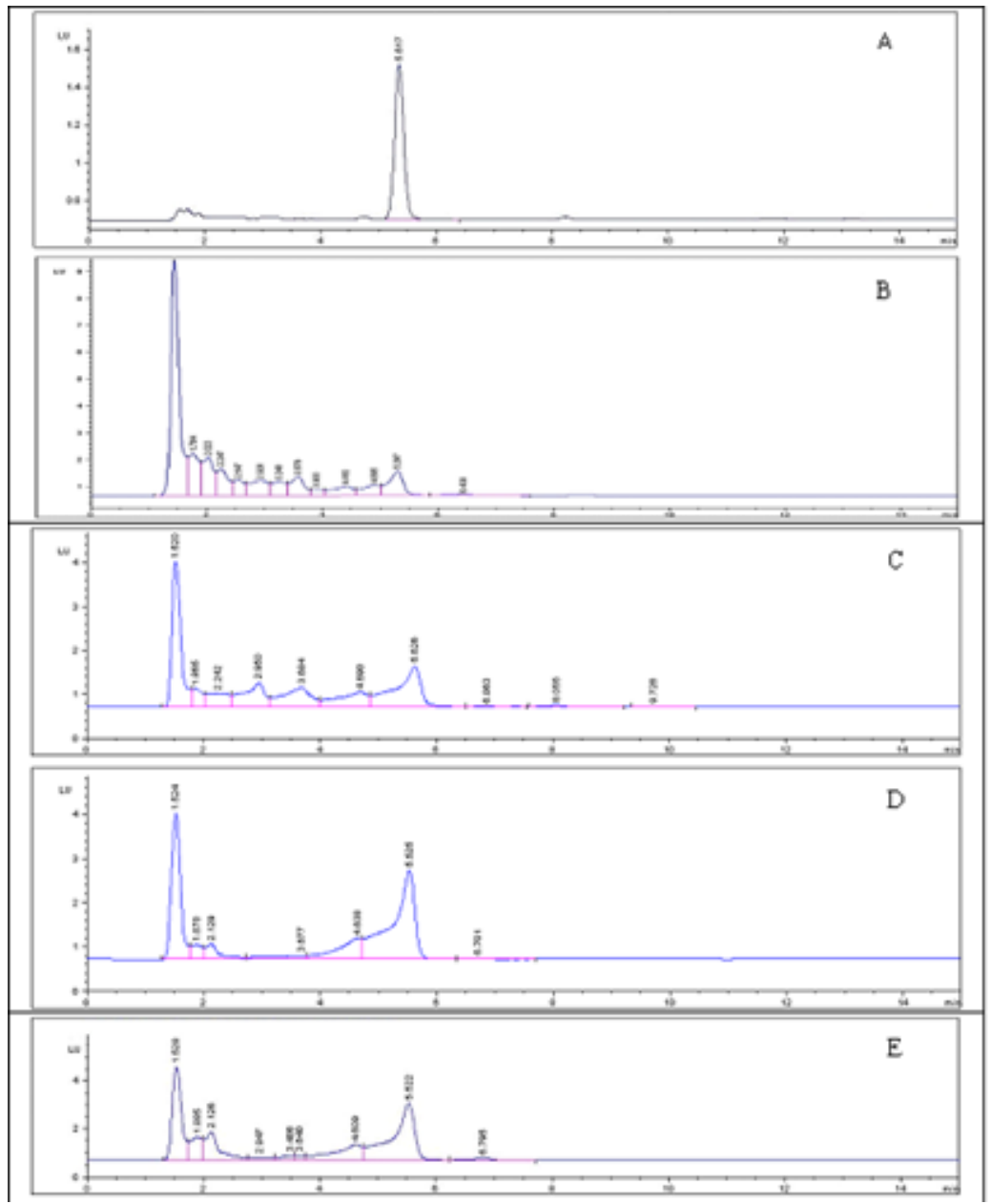

Figure 7: Chromatograms of ferulic acid, Kanji and ethanol extract of roots of Daucus carota L., A (Ferulic acid); B (Kanji at 0 month); C (Kanji at $30^{\circ} \mathrm{C}$ after 6 months); $\mathrm{D}$ (Ethanol extract at 0 month); $\mathrm{E}$ (Ethanol extract at $30^{\circ} \mathrm{C}$ after 6 months).

extract kept at different storage conditions with the passage of time are given in Tables $2 \mathrm{a}$ and $2 \mathrm{~b}$ and Figures 8 and 9 , respectively. At $30^{\circ} \mathrm{C}$ and $40^{\circ} \mathrm{C}$ the decomposition was found to be slow as compared to that of the $60^{\circ} \mathrm{C}$. The percentage contents were found to be decreasing with the increase in temperature and relative humidity. The stability trend of ferulic acid in both Kanji and ethanol extract was alike; however, it was faster in case of Kanji. These results are consistent to the previous work that indicates that decomposition increases with rise in temperature $[16,17]$. The rate of a chemical reaction increases by a factor between 2-3 times for every $10^{\circ} \mathrm{C}$ rise in temperature [15]. The results mentioned in Tables $2 \mathrm{a}$ and $2 \mathrm{~b}$ showed that the samples stored at $30^{\circ} \mathrm{C} / 65 \% \mathrm{RH}$ and at $40^{\circ} \mathrm{C} / 75 \% \mathrm{RH}$ were stable for 6 months because the decrease in concentration was less than $10 \%$. On the other hand, the products were not stable at $60^{\circ} \mathrm{C} / 85 \% \mathrm{RH}$ wherein decrease in concentration after 6 months was more than $10 \%$.

Kinetic parameters: The order of degradation or order of the reaction at different temperatures for Kanji and ethanol extract determined by graphical method was found to be zero order reaction. The plots for the determination of order of the reaction of Ferulic acid in Kanji are shown in Figure 10. The slopes of the straight lines corresponding to zero order reaction plots were taken as rate constants at different temperatures.
To predict the shelf life of Kanji and ethanol, rate constants at $25^{\circ} \mathrm{C}$ were calculated from linear regression equation of the graph $\mathrm{Ln} \mathrm{K}$ and $(1 / T)$. The graphs of Kanji and ethanol extract are given in Figure 11. The same equation was used to calculate $\mathrm{Ea}$ and pre-exponential factor (A). The activation energy and pre-exponential factor presented in Table 3 indicated that the activation energy of ethanol extract was higher as compared to kanji. A similar trend was also found in values of the pre-exponential factor of the marker in ethanol extract and kanji. These results showed that rate constants, activation energy and pre-exponential factors were increased with the rise in temperature. It was further found that stability behavior of ethanol extract based on ferulic acid contents was better than that of the Kanji. All the kinetic parameters of ethanol extract were lesser than that of the Kanji. It indicated that ethanol extract is more stable than the Kanji.

Shelf life $\left(t_{90}\right)$ : Based on the rate constants due to degradation of ferulic acid in Kanji and ethanol extract, shelf lives were calculated by dividing factor 0.105 with degradation rate constants. The estimated shelf lives of both the products at various storage conditions are presented in Table 4 . These results showed that stability was lesser at higher temperature. However, ethanol extract at all the temperatures was found to be more stable than Kanji. The possible reason of this difference may be the addition of some additional ingredients in the 


\begin{tabular}{|c|c|c|c|c|c|c|c|c|c|}
\hline $30^{\circ} \mathrm{C}$ & Area & Area & Mean & SD & $\mu \mathrm{g} / \mathrm{mL}$ & $\mathrm{mg} / \mathrm{g}$ & $\%$ & $1 n$ & $1 / C$ \\
\hline 0 & 46.56 & 46.85 & 46.705 & 0.205061 & 83.19153 & 16.63831 & 1.663831 & 2.8117011 & 0.060102 \\
\hline 1 & 46.53 & 46.72 & 46.625 & 0.13435 & 83.04793 & 16.60959 & 1.660959 & 2.80998 & 0.060206 \\
\hline 2 & 46.43 & 46.43 & 46.43 & 0 & 82.6979 & 16.53958 & 1.653953 & 2.805756 & 0.060461 \\
\hline 4 & 46.31 & 46.29 & 46.3 & 0.014142 & 82.46455 & 16.49291 & 1.619291 & 2.602931 & 0.060632 \\
\hline 6 & 45.44 & 41.311 & 45.41 & 0.042426 & 80.86699 & 16.1734 & 1.61734 & 2.783368 & 0.06183 \\
\hline \multicolumn{10}{|l|}{$40^{\circ} \mathrm{C}$} \\
\hline 0 & 46.56 & 46.85 & 46.705 & 0.205061 & 83.19153 & 16.63831 & 1.663831 & 2.811708 & 0.060102 \\
\hline 1 & 46.53 & 46.32 & 46.675 & 0.205061 & 83.13768 & 16.62754 & 1.662754 & 2.81106 & 0.060141 \\
\hline 2 & 46.52 & 46.76 & 46.64 & 0.169706 & 83.07485 & 16.61497 & 1.461497 & 2.810304 & 0.060187 \\
\hline 4 & 46.34 & 36.44 & 46.39 & 0.070711 & $82 . .6261$ & 16.52522 & 1.652522 & 2.804888 & 0.060514 \\
\hline 6 & 45.12 & 45.17 & 45.145 & 0.035355 & 80.39131 & 16.07826 & 1.607826 & 2.777468 & 0.062196 \\
\hline \multicolumn{10}{|l|}{$60^{\circ} \mathrm{C}$} \\
\hline 0 & 46.56 & 46.56 & 46.56 & 0 & 82.93125 & 16.58625 & 1.658625 & 2.808574 & 0.060291 \\
\hline 1 & 44.65 & 44.51 & 44.58 & 0.098995 & 79.37713 & 15.87543 & 1.587543 & 2.764772 & 0.06299 \\
\hline 2 & 43.87 & 42.97 & 43.42 & 0.636396 & 77.29492 & 15.45898 & $1 . .545898$ & 2.73819 & 0.064687 \\
\hline 4 & 43.08 & 43.24 & 43.16 & 0.113137 & 76.82822 & 15.36564 & 1.536564 & 2.732134 & 0.06508 \\
\hline 6 & 42.83 & 42.43 & 42.63 & 0.282843 & 75.87686 & 15.17537 & 1.517337 & 2.719674 & 0.065896 \\
\hline
\end{tabular}

Table 2a: Stability of ethanol extract of roots of Daucus Carota L. at three storage conditions.

\begin{tabular}{|c|c|c|c|c|c|c|c|c|c|}
\hline $30^{\circ} \mathrm{C}$ & Area & Area & Mean & SD & $\mu \mathrm{g} / \mathrm{ml}$ & $\mathrm{mg} / \mathrm{g}$ & $\%$ & $1 n$ & $1 / C$ \\
\hline 0 & 26.85 & 26.45 & 26.65 & 0.282843 & 51.63212 & 10.32642 & 1.032642 & 2.334706 & 0.096839 \\
\hline 1 & 26.82 & 26.44 & 26.63 & 0.268701 & 51.59024 & 10.31805 & 1.031805 & 2.333895 & 0.096918 \\
\hline 2 & 26.79 & 26.43 & 26.61 & 0.254558 & 51.54837 & 10.30967 & 1.030967 & 2.333083 & 0.096996 \\
\hline 4 & 26.77 & 25.96 & 26.365 & 0.572756 & 51.03539 & 10.20708 & 1.020708 & 2.323081 & 0.097971 \\
\hline 6 & 25.2 & 25.11 & 25.155 & 0.06364 & 48.50188 & 9.700377 & 0.970038 & 2.272165 & 0.103039 \\
\hline \multicolumn{10}{|l|}{$40^{\circ} \mathrm{C}$} \\
\hline 0 & 26.85 & 26.75 & 26.8 & 0.070711 & 51.94619 & 10.38924 & 1.038924 & 2.34077 & 0.096253 \\
\hline 1 & 26.81 & 26.72 & 26.765 & 0.06364 & 51.87291 & 10.37458 & 1.037458 & 2.339359 & 0.096389 \\
\hline 2 & 26.66 & 26.74 & 26.7 & 0.056569 & 51.73681 & 10.34736 & 1.034736 & 2.336732 & 0.096643 \\
\hline 4 & 25.61 & 25.98 & 25.795 & 0.26163 & 49.84192 & 9.968384 & 0.996838 & 2.299418 & 0.100317 \\
\hline 6 & 25.27 & 25.67 & 25.47 & 0.282843 & 49.16143 & 9.832286 & 0.983229 & 2.285672 & 0.101706 \\
\hline \multicolumn{10}{|l|}{$60^{\circ} \mathrm{C}$} \\
\hline 0 & 26.85 & 26.75 & 26.8 & 0.070711 & 51.94619 & 10.38924 & 1.038924 & 2.34077 & 0.096253 \\
\hline 1 & 25.86 & 26.76 & 26.31 & 0.636396 & 50.92023 & 10.18405 & 1.013405 & 2.320822 & 0.098193 \\
\hline 2 & 25.8 & 25.69 & 25.745 & 0.077782 & 49.73723 & 9.947446 & 0.994745 & 2.297316 & 0.100528 \\
\hline 4 & 25.05 & 25.15 & 25.1 & 0.070711 & 48.38673 & 9.677345 & 0.967735 & 2.269788 & 0.103334 \\
\hline 6 & 23.24 & 23.14 & 23.19 & 0.070711 & 44.38756 & 8.877513 & 0.887751 & 2.183521 & 0.112644 \\
\hline
\end{tabular}

Table 2b: Stability of Kanji at three storage conditions.

Kanji. These ingredients seemed to increase the degradation rate of ferulic acid. This data provides the evidence that ethanol extract of roots of black carrots can be used to prepare powdered or dispersible formulation that can be used in place of Kanji by dissolving in water.

Stability testing indicates pattern of drug degradation due to environmental factors with the passage of time [18]. The stability testing of a product needs to be conducted for a period which corresponds to the actual storage time and temperature [17]. Usually drugs are stored at room temperature, if not specified in individual monographs, and degradation is slow at this storage condition hence shelf life may go up to several years. The stability testing for such a long period is time consuming and expensive. Therefore, stability studies are conducted at high temperatures to predict long term stability within a short time.
Due to this reason, the stability of Kanji and ethanol extract of its main ingredient was investigated at accelerated conditions.

Stability is influenced by a number of physical and chemical factors. The temperature, moisture and light may cause hydrolysis, oxidation, polymerization and isomerisation. Moreover, temperature increases kinetic energy which in turn increases collision molecules. Moisture is particularly important to drugs which are susceptible to hydrolysis [19]. Moisture can also facilitate microbial growth which deteriorates constituents along with production of toxic chemicals. Drugs instability due to oxidation is nearly equivalent to that of hydrolysis. The temperature and light enhance the rate of oxidation. In the present study, it has been noted that ferulic acid contents decreased when the products were stored at elevated temperature and higher relative 


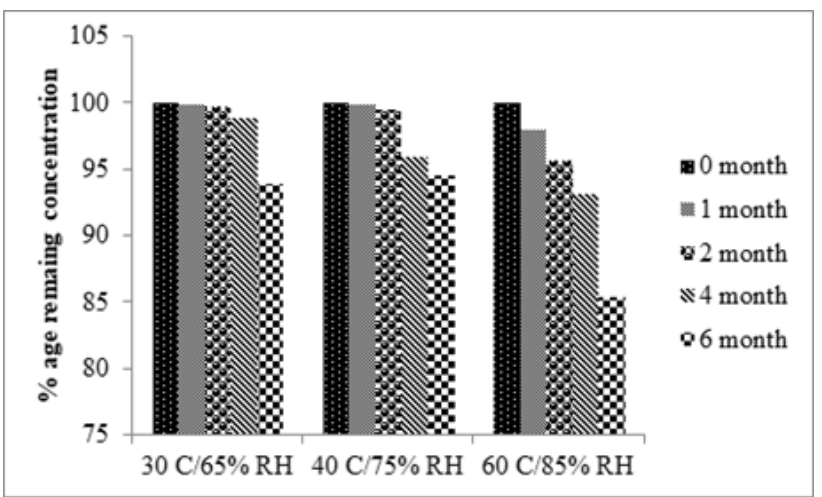

Figure 8: Stability of Kanji with reference to ferulic acid stored at $30^{\circ} \mathrm{C} / 65 \% \mathrm{RH}$, $40^{\circ} \mathrm{C} / 75 \% \mathrm{RH}$ and $60^{\circ} \mathrm{C} / 85 \% \mathrm{RH}$

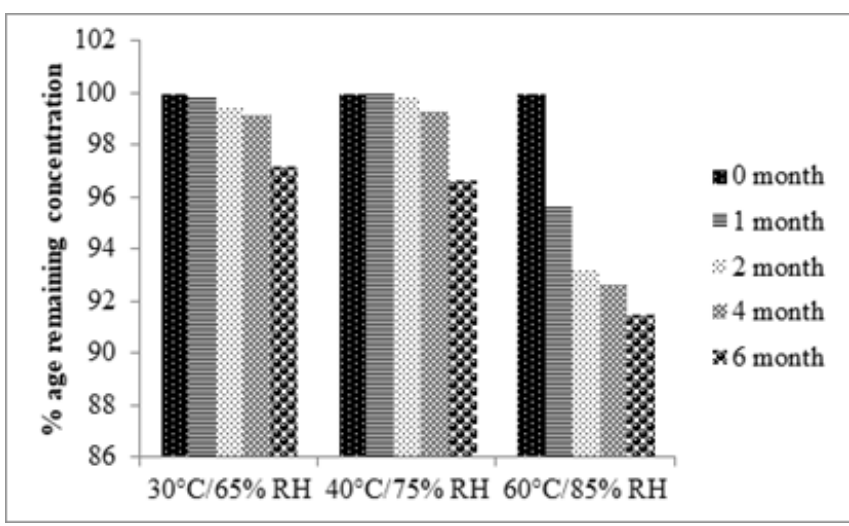

Figure 9: Stability of ethanol extracts with reference to ferulic acid at $30^{\circ} \mathrm{C} / 65 \%$ $\mathrm{RH}, 40^{\circ} \mathrm{C} / 75 \% \mathrm{RH}$ and $60^{\circ} \mathrm{C} / 85 \% \mathrm{RH}$

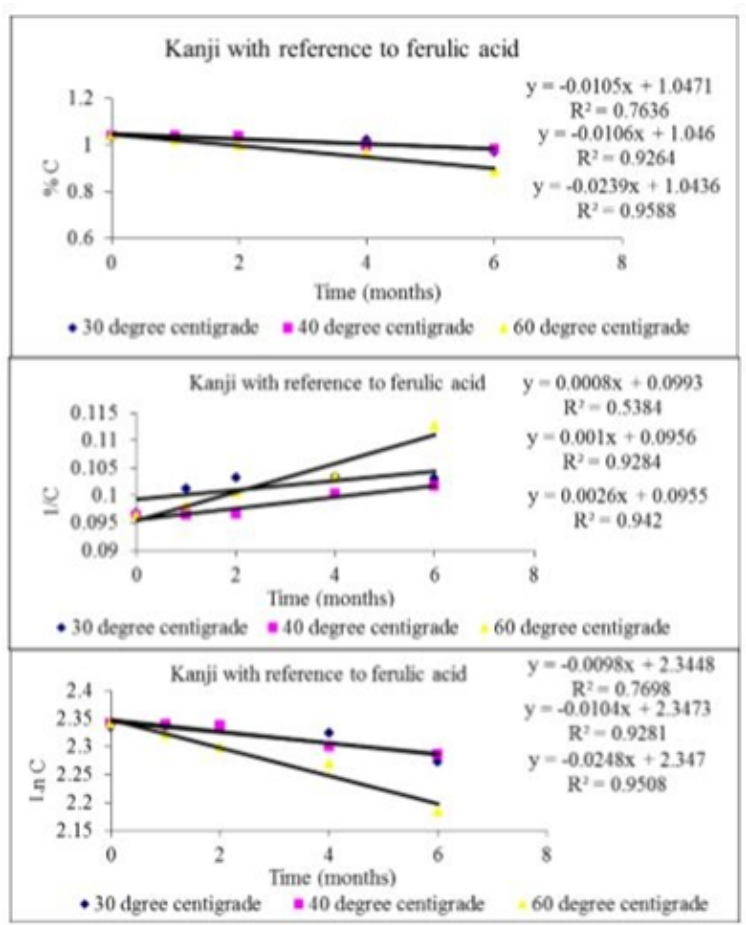

Figure 10: Plots for determination of order of reaction and rate constant $(\mathrm{K})$ of kanji with reference to ferulic acid, C (concentration), in (natural logarithm).
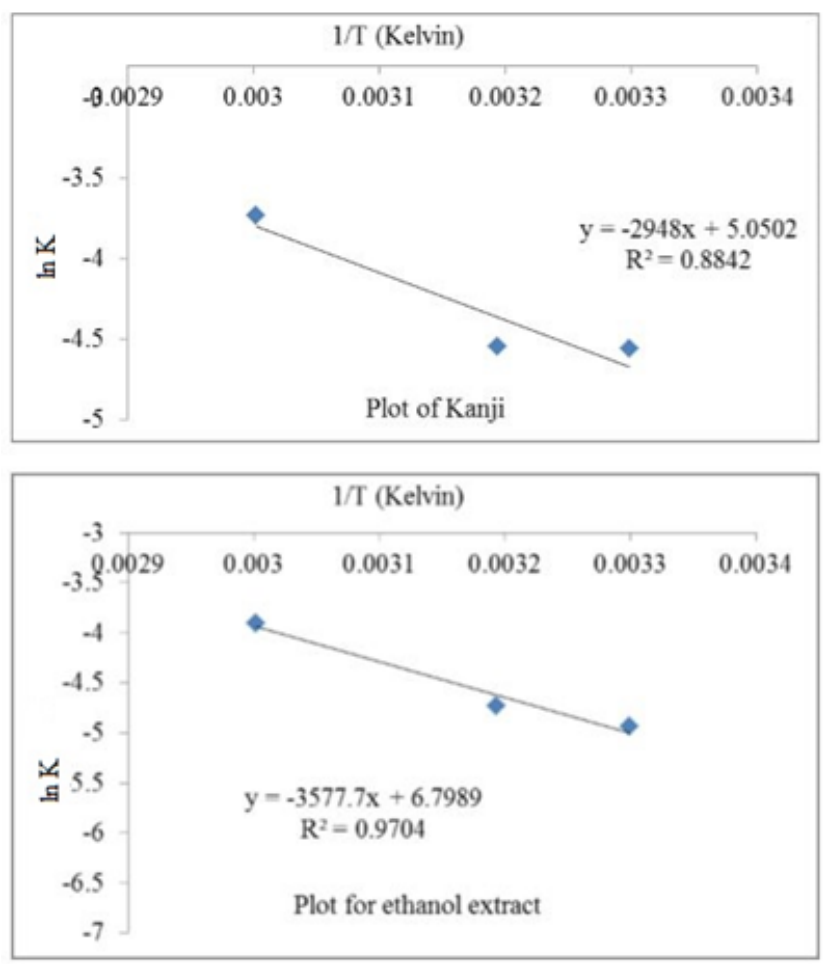

Figure 11: Plots of InK and 1/T of kanji and ethanol extract of roots of Daucus carota $\mathrm{L}$. for the prediction of rate constant, activation energy and pre-exponential factor, $\mathrm{K}$ (rate constant); in (natural logarithm).

\begin{tabular}{|c|c|c|c|c|c|c|}
\hline Ferulic acid & $\mathrm{K} 25^{\circ} \mathrm{C}$ & $\mathrm{K} 30^{\circ} \mathrm{C}$ & $\mathrm{K} 40^{\circ} \mathrm{C}$ & $\mathrm{K} 60^{\circ} \mathrm{C}$ & $\begin{array}{c}\mathrm{Ea}\left(\mathrm{J} \mathrm{mol}^{-}\right. \\
1) 25^{\circ} \mathrm{C}\end{array}$ & $A\left(S^{-1}\right)$ \\
\hline Kanji & 0.0081 & 0.0105 & 0.0106 & 0.0239 & 24509.76 & 156.053 \\
\hline $\begin{array}{c}\text { Ethanol } \\
\text { extract }\end{array}$ & 0.0056 & 0.0072 & 0.0088 & 0.0202 & 29745.00 & 896.86 \\
\hline
\end{tabular}

Table 3: Rate constant (K), activation energy (Ea) and pre-exponential factor $(\mathrm{A})$ of Kanji and ethanol extract of Daucus carota L. with reference to ferulic at different temperatures.

\begin{tabular}{|c|c|c|c|c|}
\hline Ferulic acid & $\mathbf{t}_{\mathbf{9 0}}$ & $\mathbf{t}_{\mathbf{9 0}}$ & $\mathbf{t}_{\mathbf{9 0}}$ & $\mathbf{t}_{\mathbf{9 0}}$ \\
\hline $\mathbf{2 5}^{\circ} \mathbf{C}$ & $\mathbf{3 0}^{\circ} \mathbf{C} / \mathbf{6 0} \% \mathbf{R H}$ & $\mathbf{4 0}^{\circ} \mathbf{C} / \mathbf{7 5} \% \mathbf{R H}$ & $\mathbf{6 0}^{\circ} \mathbf{C} / \mathbf{8 5} \% \mathbf{R H}$ \\
\hline $\begin{array}{c}\text { Kanji } \\
\text { extranol }\end{array}$ & 12.96 & 10.00 & 9.90 & 4.39 \\
\hline
\end{tabular}

Table 4: Shelf life $\left(t_{90}\right)$ of Kanji and ethanol extract of Daucus carota L. with reference to ferulic at different storage conditions.

humidity. The kinetic parameters of Kanji were higher than that of ethanol extract of roots of the plant. The ethanol extract was not having additional ingredients such as table salt, chilies and mustard seed that is why it was found to be having higher shelf life. This indicates that ethanol extract can be used to prepare solid dosage forms which can be made available for a longer period of time.

\section{Conclusion}

UV-Visible fingerprint profiles of Kanji and ethanol extract stored at different storage conditions exhibited visual changes in the fingerprints. The samples stored at $60^{\circ} \mathrm{C} / 85 \% \mathrm{RH}$ for six months have shown apparent changes in spectrum. It means that both Kanji and ethanol extract deteriorated at high temperature with the passage of time. 
Citation: Latif A, Hussain K, Bukhari NI, Shafi H, Mazhar M (2018) Metabolomics and Marker Based Accelerated Stability of Kanji and Ethanol Extract of its Main Ingredient, Daucus carota L. Roots. Pharm Anal Acta 9: 589. doi: 10.4172/2153-2435.1000589

Page 8 of 8

Analysis of Kanji and ethanol extract by HPLC indicated that the marker compound in both the samples followed the zero-order reaction. It means that rate of degradation was not dependent on initial concentration of the ferulic acid. The results of the study indicate that ethanol extract is more stable than that of the Kanji.

\section{References}

1. Achal K, Prasad NAV, Laddha KS (2008) Stability testing of herbal products. Pharm Rev 109

2. Heinrich M (2007) HerbalGram. 76: 62-64.

3. Li S, Han Q, Qiao C, Song J, Cheng CL, et al. (2008) Chemical markers for the quality control of herbal medicines: an overview. Chinese Med 3: 7.

4. http://www.ema.europa.eu/docs/en_GB/document_library/Scientific_ guideline/2009/09/WC500002651.pdf

5. Baloch AF (1994) Vegetable crops. Horticulture 500.

6. https://www.jcsp.org.pk/PublishedVersion/f0be61b7-dda1-48eb-bc3caad660d51cfd 1st\%20gally\%20prof\%20fo\%209576\%20_KHALID\%20 HUSSAIN_.doc\%20checked.pdf

7. Berry SK, Sehgal RC, Kalra CL (1999) Comparative oil uptake by potato chips during frying under different conditions. J Food Sci Tech 36: 519-521.

8. Sahota P, Pandove G, Jairath S, Banta G (2008) A functional probiotic beverage: Kanji. Indian J Ecol 35: 101-102.

9. EMEA (2001) Final proposals for revision of the notes for guidance on quality of herbal medicinal products. The European Agency for the Evaluation of Medicinal Products.

10. Young JF (1967) Humidity control in the laboratory using salt solutions-a review.

\section{J Appl Chem 17: 241-245.}

11. Greenspan L (1977) Humidity fixed points of binary saturated aqueous solutions. J Res National Bureau Standards 81: 89-96.

12. Marsh KN, Marsh KN (1987) Recommended reference materials for the realization of physicochemical properties. Oxford: Blackwell Scientific Publications.

13. ASTM (1991) Standard practice for maintaining constant relative humidity by means of aqueous solution. In: Annual Book of ASTM Standards: Designation. Am Soc Testing Materials.

14. Murphy B, Murphy C, Hathaway BJ (1997) A working method approach for introductory physical chemistry calculations: numerical and graphical problem solving. Royal Society of Chemistry, Cambridge, UK

15. Pugh J (2002) Kinetics and Product Stability. Churchill Livingstone, London.

16. Pourrat H, Barthomeuf C, Pourrat A, Cottier PE, Ibrahim H (1995) Stabilization of Octastation, a Somatostatin Analogue. Preparation of freeze-dried products for parenteral injection. Biol Pharmaceutical Bull 18: 766-771.

17. Khalid H, Zhari I, Amirin S, Pazilah I (2011) Accelerated stability and chemical kinetics of ethanol extracts of fruit of piper sarmentosum using high performance liquid chromatography. Iran J Pharm Res 10: 403.

18. WHO (1996) WHO expert committee on specifications for pharmaceutical preparation annex 5- guidelines for stability testing of pharmaceutical products containing well-established drug substances in conventional dosage forms. WHO Technical Report Series, No. 863. WHO, Geneva.

19. Waterman KC, Adami RC, Alsante KM, Antipas AS, Arenson DR, et al. (2002) Hydrolysis in pharmaceutical formulations. Pharmaceutical Dev Technol 7: 113 146. 